\title{
Study of the Method of Multi-Frequency Signal Detection Based on the Adaptive Stochastic Resonance
}

\author{
Zhenyu Lu, ${ }^{1}$ Tingya Yang, ${ }^{2}$ and Min Zhu ${ }^{3}$ \\ ${ }^{1}$ College of Electrical and Information Engineering, Nanjing University of Information Science and Technology, Nanjing 210044, China \\ ${ }^{2}$ Jiangsu Meteorological Observatory, Nanjing 210008, China \\ ${ }^{3}$ College of Information and Control, Nanjing University of Information Science and Technology, Nanjing 210044, China
}

Correspondence should be addressed to Zhenyu Lu; luzhenyu76@163.com

Received 1 January 2013; Accepted 12 March 2013

Academic Editor: Xuerong Mao

Copyright (C) 2013 Zhenyu Lu et al. This is an open access article distributed under the Creative Commons Attribution License, which permits unrestricted use, distribution, and reproduction in any medium, provided the original work is properly cited.

\begin{abstract}
Recently, the stochastic resonance effect has been widely used by the method of discovering and extracting weak periodic signals from strong noise through the stochastic resonance effect. The detection of the single-frequency weak signals by using stochastic resonance effect is widely used. However, the detection methods of the multifrequency weak signals need to be researched. According to the different frequency input signals of a given system, this paper puts forward a detection method of multifrequency signal by using adaptive stochastic resonance, which analyzed the frequency characteristics and the parallel number of the input signals, adjusted system parameters automatically to the low frequency signals in the fixed step size, and then measured the stochastic resonance phenomenon based on the frequency of the periodic signals to select the most appropriate indicators in the middle or high frequency. Finally, the optimized system parameters are founded and the frequency of the given signals is extracted in the frequency domain of the stochastic resonance output signals. Compared with the traditional detection methods, the method in this paper not only improves the work efficiency but also makes it more accurate by using the color noise, the frequency is more accurate being extracted from the measured signal. The consistency between the simulation results and analysis shows that this method is effective and feasible.
\end{abstract}

\section{Introduction}

Now, we need to find and extract useful signal through the signal detection in engineering technology and scientific research. The traditional method to detect signal usually uses linear filtering, wavelet analysis [1], and so on to reduce and eliminate noise and finally obtain the useful signal. Although some weak signals are often overwhelmed by strong noise, the weak periodic signal is also reduced in the denoise to a certain extent, which made some weak periodic signal fail to be detected and extracted. In 1981, Benzi et al. proposed the concept of stochastic resonance [2] which provides a new research method for the detection of weak periodic signal. Compared to the traditional signal detection method, stochastic resonance is a kind of nonlinear phenomenon, which adds a certain intensity noise rather than reducs the noise, then uses the synergy among signal frequency, noise intensity, and nonlinear system to drive part of the noise energy into the measuring signal energy, and finally highlights in the output signal.

With the development of the theory of stochastic resonance, the method of finding and extracting weak periodic signals from strong noise by stochastic resonance effect has been widely used in various fields of science such as nerve physiology, intelligence theory, nonlinear optics, signal processing, communication engineering, and sociology [3-11]. Among them, the method of detecting single-frequency weak signals by using stochastic resonance effect has been more mature. Its main method is to analyze the relationship between the characteristics of the measured input signal and the system parameters through the nonlinear bistable system, through adjusting the system parameters [12] or increasing the strength of the noise $[13,14]$ to realize stochastic resonance. In 1990, Gang et al. [15] put forward the famous idea of adiabatic approximation theory, which proved that stochastic resonance is used to detect small parameter signal. 
Then the method of stochastic resonance detection to singlefrequency signal is gradually perfect. However, in the actual research, we found that the signal submerged by strong noise is unknown weak periodic signal and even unknown high frequency signal. Then, the research on the detection of multiple frequency signals received the widespread attention rapidly.

It is mainly used to realize stochastic resonance through adjusting system parameters manually or increasing the strength of noise so that we can find and extract the unknown multiple frequency signal. Due to the manual, adjusting has low work efficiency, and cannot achieve continuous search which will omit part of the signal, and it is difficult to find and search the optimal system parameters which will certainly omit part of the signal. This paper combines the theory of stochastic resonance and adaptive algorithm to put forward a kind of adaptive stochastic resonance detection method for multiple-frequency signal, respectively, of the low frequency and high frequency input signals. Based on the traditional single-frequency weak signal detection, selected the SNR to be a measurement index of the generation of stochastic resonance and reducing the range of parameter values by the threshold analysis, this method can find the optimal system parameters effectively and can detect a multiple weak periodic signals. A large number of simulation results show that the output signal of stochastic resonance system will be interfered by some noise which will lead to distortion of waveform slightly. Therefore, this paper makes processing the output signal of stochastic resonance by using the autocorrelation method which only changes the amplitude and phase, without changing the frequency. It can reduce the impact of noise, make the waveform more similar to measured signal, highlight the frequency of the signal cycle component, and enhance the SNR.

The methods to detect the high-frequency signals are subsampled, frequency-shifted and rescaling, wavelet analysis $[16,17]$, and so forth. Its main idea is transforming the high frequency into the low frequency through scale changes to meet the conditions of stochastic resonance then detect and extract the low-frequency signal, and finally achieve recovery. However, the output signal waveform extracted by these methods often exists with some distortion. In 2008, Mao et al. [18] proposed a method, which adds one cycle modulated signal to the stochastic resonance system, and then adjust the frequency of the modulation signal close to the frequency of the signal to be measured and generate the differential frequency which meets the adiabatic approximation theory. Finally, significant changes of the output signal spectrogram occurred in the approximation process. This characteristic can be taken as the basis for signal detection and extraction. But it used ideal Gaussian white noise during the experiment rather than the nonzero color noise which is often encountered in practical engineering applications such as the mechanical fault detection [8], and its frequency is concentrated in a frequency band and can easily be confused with the frequency of signal to be measured. It is considered that the frequency of the multi-frequency signal to be measured may be odd multiples. This paper contemplated to select the reciprocal of the power spectrum in the autocorrelation

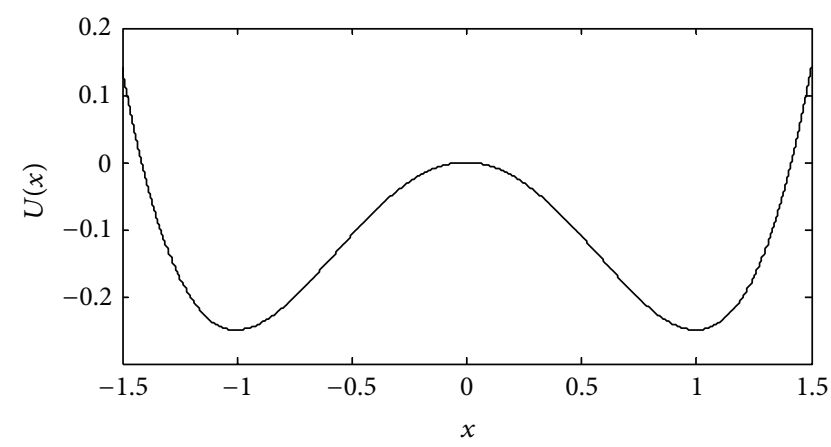

FIgURE 1: When $A=0, D=0$, the corresponding potential function curve $U(x)$.

function of the output signal as measurement index under the interference of the color noise, which can distinguish the color noise with the signal to be measured and extract the high frequency of multiple parallel input signals effectively. This paper made a large number of numerical simulations by MATLAB, and the simulation results show the effectiveness and feasibility of the method and have a good prospect.

\section{Bistable System and Its Performance Analysis}

This paper uses the bistable system model: Langevin equation. It is actually an overdamped bistable system model driven by cycle, and its mathematical expression is [19]

$$
\frac{d x}{d t}=a x-b x^{3}+s(t)+\Gamma(t),
$$

where $a, b$ are the system parameters, $s(t)$ is the system input signal to be measured, $s(t)=A \cos \left(2 \pi f_{0} t\right), f_{0}$ is the frequency of the input signal to be measured and $\Gamma(t)$ is the Gaussian white noise with noise intensity $D$, and it satisfied: $\langle\Gamma(t)\rangle=0$, $\left\langle\Gamma(t) \Gamma\left(t^{\prime}\right)\right\rangle=2 D \delta\left(t-t^{\prime}\right)$. When the input signal $A=0$, the noise intensity $D=0$, the potential function corresponding to the nonlinear bistable system is

$$
U(x)=-\frac{1}{2} a x^{3}+\frac{1}{4} b x^{3} .
$$

As shown in Figure 1, the system has two potential wells and a potential barrier. Stochastic resonance is actually shown the phenomenon that the signal has enough energy to transfer between two potential wells under the synergistic effect of the bistable system. At present, the main method is adjusted system parameters and increased a certain intensity of noise to generate stochastic resonance. However, the characteristic of input signal to be measured with noise is usually unknown in the measurement of the practical engineering. It is difficult to meet the actual demand only by adjusting the system parameters manually. Therefore, this paper integrates the adaptive iterative algorithm into the stochastic resonance detection method to study the adaptive stochastic resonance detection method for multi-frequency signals, seeks the optimal system parameters to generate stochastic resonance, 
and finally finds and extracts the frequency of unknown weak cycle signal in the frequency domain.

\section{Adaptive Stochastic Resonance Detection for Low-Frequency Signals}

3.1. Measurement Index and Iterative Algorithm. Adaptive stochastic resonance signal detection involves two important factors: measurement index and iterative algorithm.

(1) Measurement Index. Selecting the appropriate measurement index to measure the effectiveness of the system output which means whether to generate stochastic resonance. The commonly measurement index in the study of stochastic resonance contains signal-to-noise ratio (SNR), autocorrelation function, cross-correlation function, mutual information, residence time distribution, [20-23] and so on. For the detection of low-frequency signals, this paper is mainly based on the SNR to extract effective signal. SNR is an index of the proportion that the energy of input signal frequency $f_{0}$ is contained in the system output signal $y(t)=g(x(t))$, which is defined as

$$
\mathrm{SNR}=10 \log \frac{S}{N}=10 \log \frac{S\left(f_{0}\right)}{N\left(f_{0}\right)} d B .
$$

This paper uses the fourth-order Runge-Kutta method to solve the nonlinear systems. Set the sample step $h=1 / f_{s}$, where $f_{s}$ is the sampling frequency. The output signal is $y(t)$. The power spectrum of the input signal $S\left(f_{0}\right)$ is the energy of the output signal power spectrum $Y(f)$ in the input signal at the frequency $f_{0}$. The noise power spectrum $N\left(f_{0}\right)$ is a period of average power spectrum estimate near the input signal frequency $f_{0}$.

(2) Iterative Algorithm. Choose a suitable iterative algorithm to make the system tends to the optimal state, which generates stochastic resonance. In the measurement of the practical engineering, by the limit of the algorithm accuracy requirements and working conditions, many algorithms cannot be applied to the actual detection because of its high complexity. This paper mainly uses adaptive iterative algorithm: fix the step size and adjust the system parameters linearity. The steps of adaptive stochastic resonance detection of low-frequency signal are as follows.

(a) Firstly, to set the system parameters, to input the signal to be measured with noise, to fix the step size, and to select the appropriate value range of parameter, increase the step size during this interval gradually to adjust the system parameters $a$.

(b) Secondly, to use the Runge-Kutta algorithm to take numerical simulation to the corresponding system of each parameter, every parameter $a$ has a corresponding system output signal.

(c) Then, to calculate the SNR according to (3), find the optimal parameters $a_{\text {best }}$ corresponding to the maximum SNR. (d) Finally, to reset nonlinear bistable system based on the optimal parameters to drive the signal to be measured with noise, generate stochastic resonance in this system. The output signal can show the signal to be measured to the greatest extent. The frequency corresponding to the spectrum peak in the spectrum diagram of the output signal is the frequency of the signal to be measured.

3.2. Simulation of Single Weak Signal Detection. Let the input signal to be tested is $S(t)=A \sin \left(2 \pi f_{0} t\right)$, in which $A=0.8$, $f_{0}=0.03 \mathrm{~Hz}$, the noise intensity $D=0.6$, the sampling frequency $f_{s}=5 \mathrm{~Hz}$. Figure 2(b) shows that the input signal to be measured has been completely submerged by the noise at this time, the parameter of bistable system $b=1$ is fixed. But it has a problem which is how to set the range of values about the system parameter $a$.

Let the input signal be a constant $A$ and the noise intensity $D=0$ (without considering the noise). The barrier of the bistable system exists with a static threshold condition: $A_{c}=\sqrt{4 a^{3} / 27 b}$. Thus we can calculate a system parameter threshold $a=1.1$ according to the above conditions of the system. Set the adjustment range of system parameters as $[1.1,5]$ and the step size $h=1 / f_{s}=0.2$. According to the adaptive iterative algorithm mentioned above, we can obtain the variation curve of SNR as the system parameter changes in Figure 3. The maximum $\mathrm{SNR}_{\max }=0.0609$, and the corresponding optimal system parameters $a_{\text {best }}=1.2$. Reset system parameters and the system obviously generated stochastic resonance effect, as shown in Figure 2(c). Although there is still some noise in the output signal, but the noise energy is significantly weakened, and it has been fully utilized and transformed into the energy of the signal to be measured. Figure 2(d) is a spectrum diagram of the output signal, when $f=0.03 \mathrm{~Hz}$ there is a very clear and sharp spectral peak.

However, the frequency of low-frequency signal is prominent by the processing of the stochastic resonance system and is easy to be extracted. Although, as the Figure 2(c) shows that the time domain diagram of output signal is still interfered by part of the noise, there are some glitches. In order to solve this problem, this paper uses the autocorrelation techniques on the postprocessing program.

Define the autocorrelation function of the signal $x(t)$ as follows:

$$
R_{x}(\tau)=\lim _{T \rightarrow \infty} \frac{1}{T} x(t) x(t+\tau) d t,
$$

where $T$ is the observation time of the signal $x(t)$, and $R_{x}(\tau)$ describes the correlation between the signal $x(t)$ and $x(t+\tau)$, due to the actual observation time $T$ is limited. Therefore define the autocorrelation function is,

$$
\widehat{R}_{x}(\tau)=\lim _{T \rightarrow \infty} \frac{1}{T} \int_{0}^{T-\tau} x(t) x(t+\tau) d t .
$$

The signals to be measured with noise are as follows:

$$
S_{n}(t)=s(t)+\Gamma(t)=A \cos \left(2 \pi f_{0} t\right)+\Gamma(t) .
$$




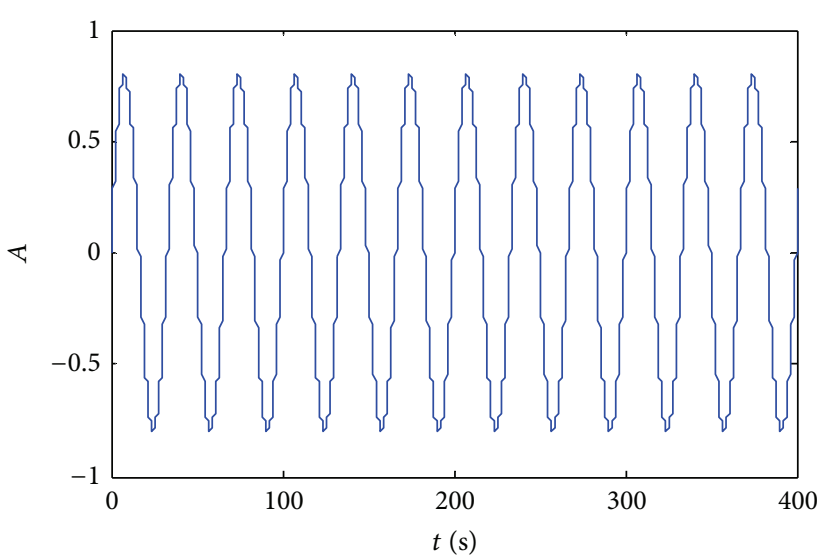

(a)

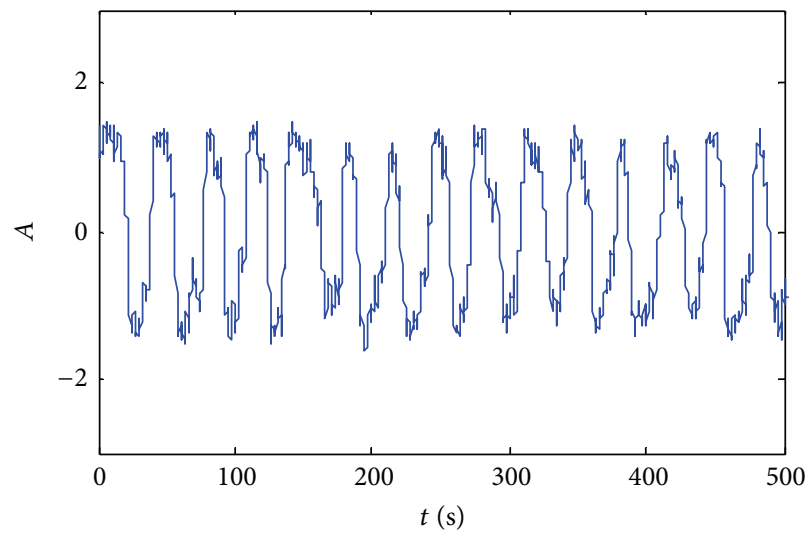

(c)

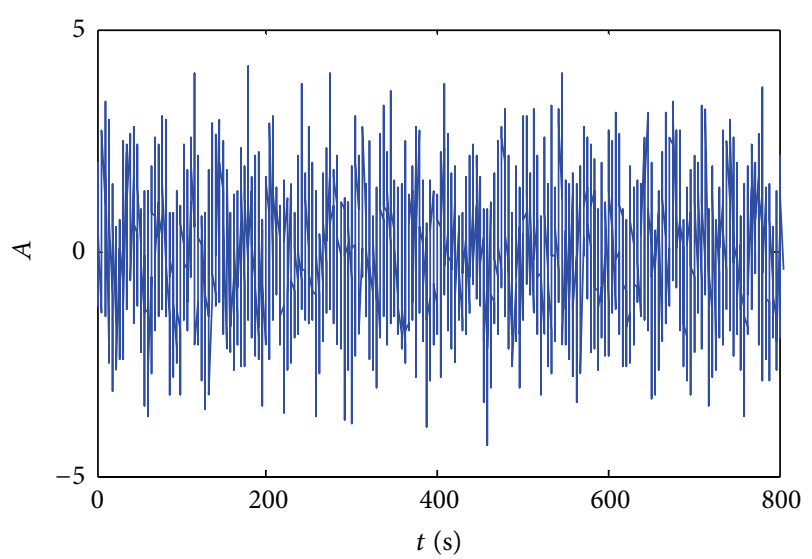

(b)

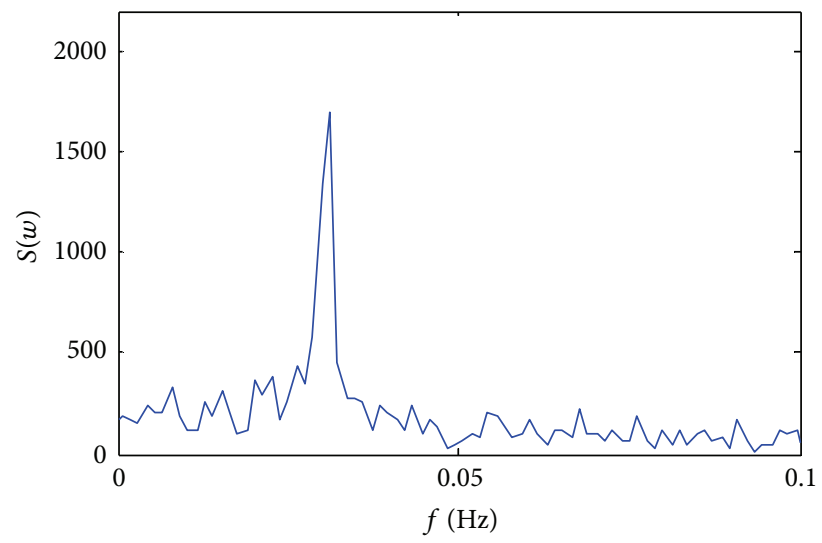

(d)

Figure 2: (a) The input signal to be measured. (b) The input signal to be measured contains white Gaussian noise. (c) The stochastic resonance output signal. (d) The spectrum figure of the stochastic resonance output signal.

For the actual engineering signal, the integration time can be approximated by $T$ instead of $T-\tau$, and the signal after the autocorrelation processing is:

$$
\begin{aligned}
R_{Y}(\tau)= & \frac{A^{2}}{2} \cos (\omega t)+\frac{A^{2}}{2 T} \int_{0}^{T} \cos [\omega(2 t+\tau)+2 \phi] d t \\
& +\frac{1}{T} \int_{0}^{T} s(t) d t \cdot \frac{1}{T} \int_{0}^{T} \Gamma(t+\tau) d t \\
& +\frac{1}{T} \int_{0}^{T} s(t+\tau) d t \cdot \frac{1}{T} \int_{0}^{T} \Gamma(t) d t+R_{\Gamma}(\tau),
\end{aligned}
$$

in which $R_{x}(\tau)$ is the autocorrelation function of the noise. The noise cannot be the ideal Gaussian white noise in the measurement of the actual engineering. Therefore, $R_{x}(\tau)$ is always present and its amplitude is drastically reduced compared with the original noise amplitude, and can be regarded as a new noise.

The output signal by autocorrelation processing can be abbreviated as

$$
y_{1}(t)=A_{1} \cos \left(f_{1} t+\phi_{1}\right)+\Gamma_{1}(t) .
$$

Compared to the original noise signal to be measured, the amplitude and phase of the two signals have changed, but the

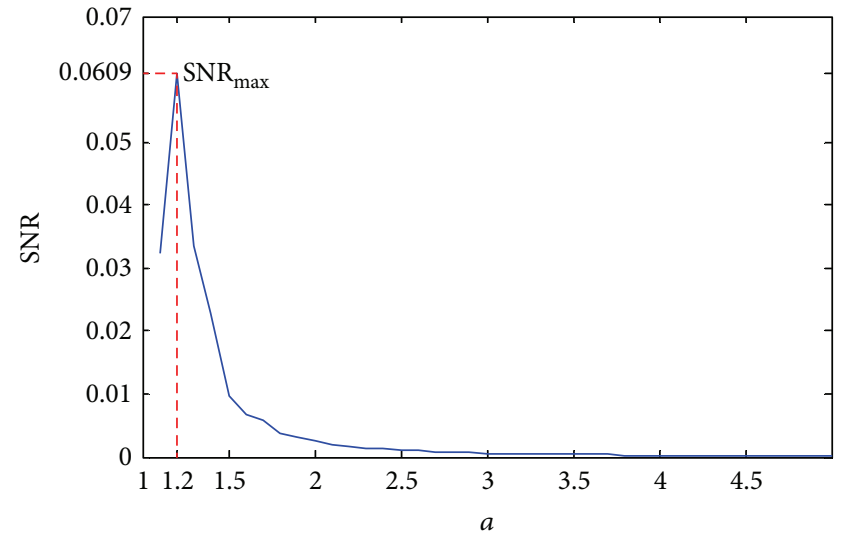

FIGURE 3: The variation curve of SNR while adjusting the system parameter $a$.

frequency is not changed. It improves the SNR to a certain extent. Therefore, this paper takes advantage of this feature to postprocess the output signal of stochastic resonance (see Figure 7). It not only reduces the influence of the noise but also makes the waveform of the output signal more close to the original signal to be measured in the time domain. 


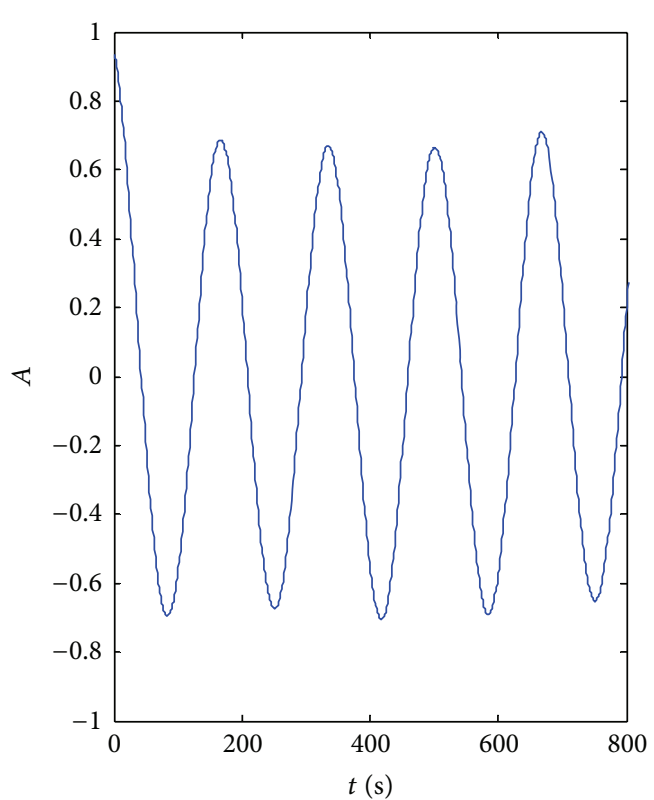

(a)

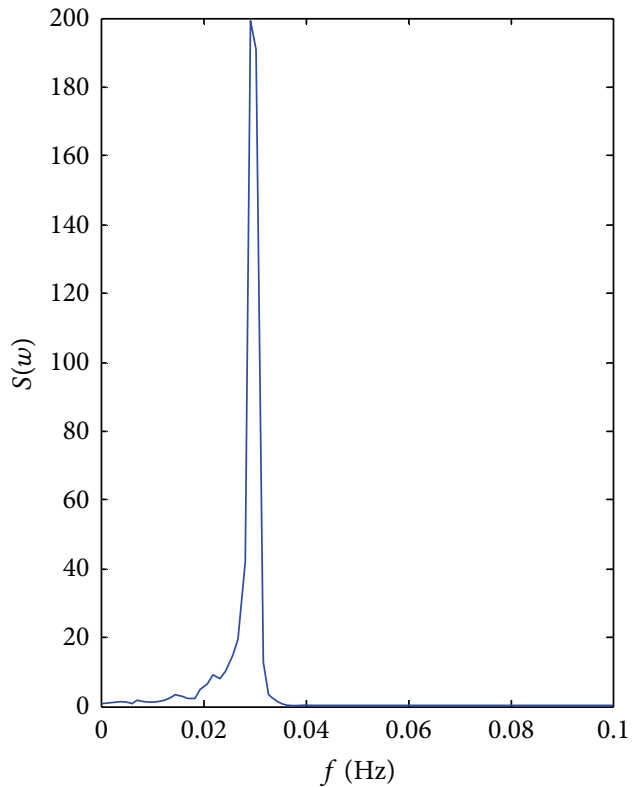

(b)

FIGURE 4: (a) The time-domain diagram of stochastic resonance output signal after correlation processing and (b) the spectrum diagram of stochastic resonance output signal after correlation processing.

With the signal cycle components characteristic frequency is even more pronounced in the spectrogram. We verify the feasibility of this theory through a numerical example. Make autocorrelation processing of the output signal of stochastic resonance as shown in Figure 2(c). As Figure 4 shows that the waveform of the output signal is obviously undistorted in the time-domain diagram, and it is almost unanimous with the waveform of the measured signal. The frequency of the signal to be measured is more prominent under the background of noise.

3.3. Simulation of Multifrequency Weak Superposition Signal Detection. When the input signal to be measured is the multi-frequency weak signal and parallel input, the multifrequency input signal to be tested is

$$
s(t)=\sum_{i=1}^{3} A_{i} \cos \left(2 \pi f_{i} t\right) .
$$

While $A_{1}=0.6, A_{2}=0.8, A_{3}=1.0, f_{1}=0.02 \mathrm{~Hz}$, $f_{2}=0.03 \mathrm{~Hz}$, and $f_{3}=0.05 \mathrm{~Hz}, \Gamma(t)$ is Gaussian white noise with noise intensity $D=0.6$. Sampling frequency $f_{s}=5 \mathrm{~Hz}$, and let the bistable system parameter $b=1$. The study has shown that only the frequency, noise intensity, and system parameters of signal must be matched, and the system can generate stochastic resonance effect, so that we define a set of system parameters as a signal path for the system [22]. It generates mixing phenomenon when the signal band is too close, and the spectrum peaks of output signal are not obvious. Therefore, we can define the frequency number as not only the channel capacity of the signal path adapts to this set of parameters to generate a stochastic resonance effect, but also the mixing frequency phenomenon does not occur. Similarly, according to the above adaptive iterative algorithm, we can calculate the optimal parameters $a_{\text {best }}=1.5$ while SNR is maximum $\left(\mathrm{SNR}_{\max }\right)$, as shown in Figure 6. As shown in Figure 5(d), the frequency of obviously spectral peak is $0.02 \mathrm{~Hz}, 0.03 \mathrm{~Hz}$, and $0.05 \mathrm{~Hz}$. The degree of waveform distortion is weakened by autocorrelation processing, and the frequency of the signal to be measured is more prominent which indicates that this algorithm is suitable for the parallel multi-frequency weak input signal detection. Parameter $a_{\text {best }}$ matches the frequency of signal to be measured and noise intensity. The channel capacity is $N=3$ at this time.

\section{Adaptive Stochastic Resonance in the High Frequency Signal Detection}

According to (1), the power spectrum of the system output signal can be calculated as [23]

$$
\begin{aligned}
S(f)= & S_{1}(f)+S_{2}(f) \\
= & \frac{2 a^{4} A^{2} \exp \left(\left(-a^{2} / 2 D\right) / \pi D^{2}\right)}{\left(2 a^{2} \exp \left(-a^{2} / 2 D\right) / \pi^{2}\right)^{2}} \times \delta\left(f_{0}-f\right) \\
& +\left[1-\frac{2 a^{4} A^{2} \exp \left(\left(-a^{2} / 2 D\right) / \pi D^{2}\right)}{\left(\left(2 a^{2} \exp \left(-a^{2} / 2 D\right) / \pi^{2}\right)+2 \pi f_{0}\right)^{2}}\right] \\
& \times\left[\frac{4 \sqrt{2} a^{4} \exp \left(\left(-a^{2} / 4 D\right) / \pi\right)}{\left(\left(2 a^{2} \exp \left(-a^{2} / 2 D\right) / \pi^{2}\right)+2 \pi f_{0}\right)^{2}}\right] .
\end{aligned}
$$

Stochastic resonance of the output signal spectrum is caused by the input signal and noise, as $S_{1}(f)$ and $S_{2}(f)$, 


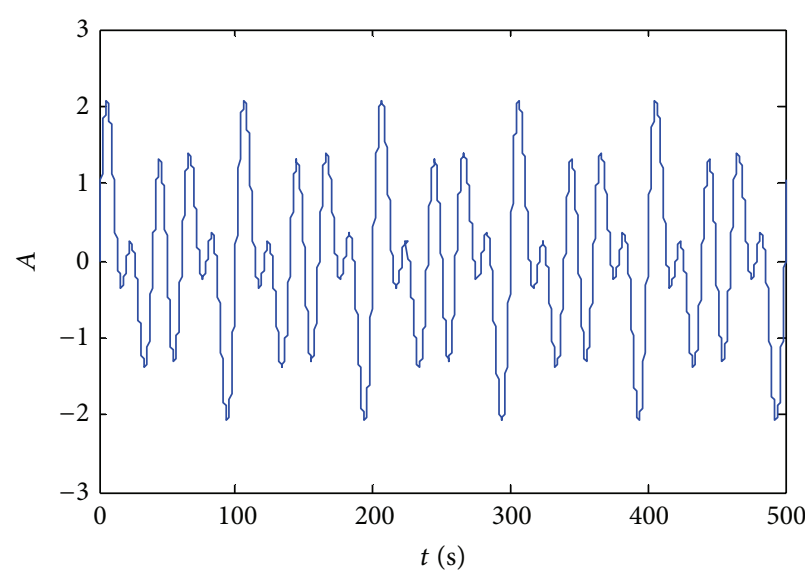

(a)

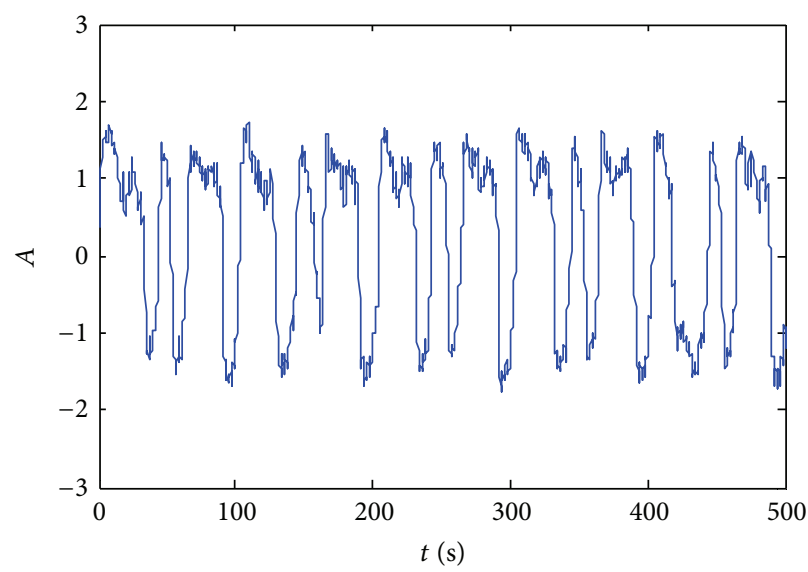

(c)

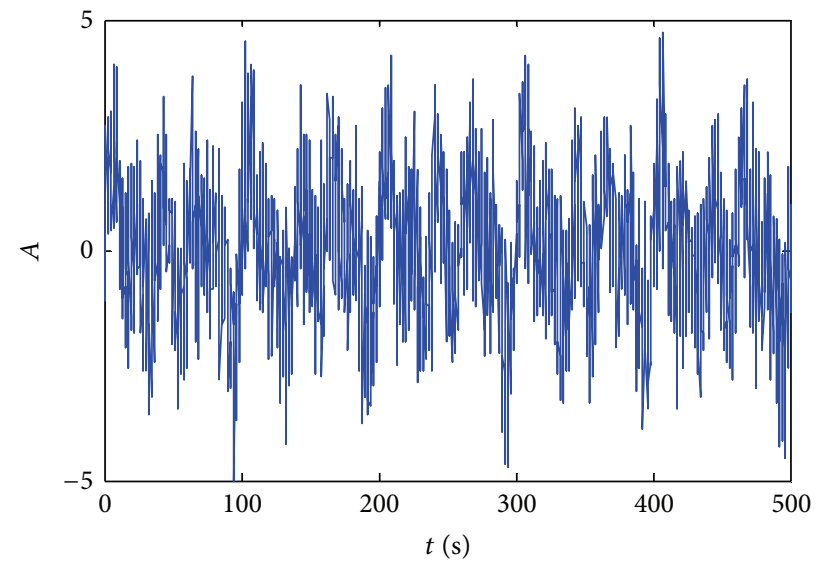

(b)

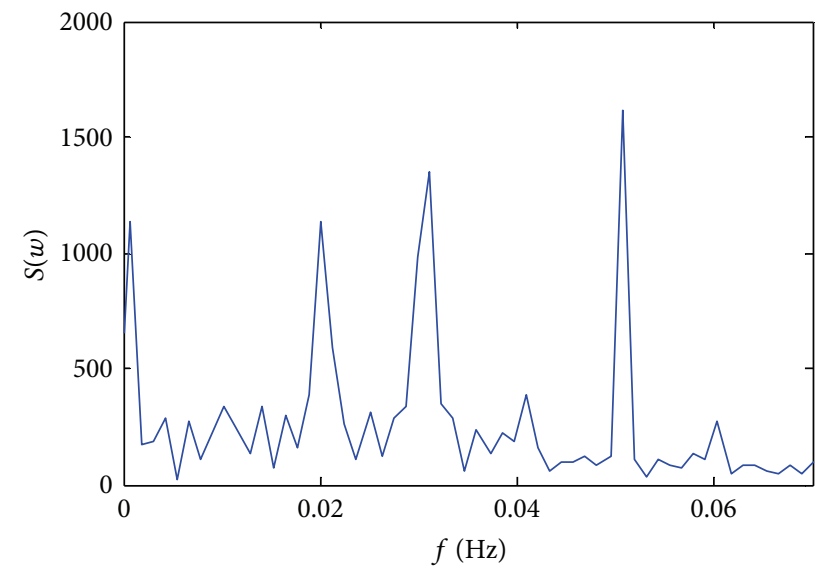

(d)

FIGURE 5: (a) The multi-frequency input signal to be measured. (b) The multi-frequency input signal to be measured contains white Gaussian noise. (c) The stochastic resonance output signal. (d) The spectrum figure of the stochastic resonance output signal.

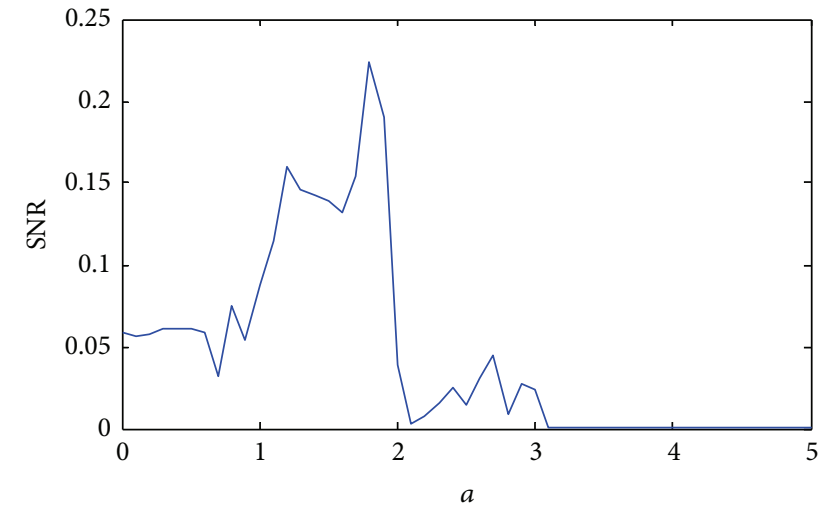

FIGURE 6: The variation curve of SNR while adjusting the system parameter $a$.

respectively. Since the output of the noise power spectrum $S_{2}(f)$ has Lorentz distribution, the subband which can generate stochastic resonance spectrum peak is generally limited to the low frequency band. Therefore, the bistable system of stochastic resonance is generally suitable for small parameters $(f \ll 1)$ of weak signal detection. For the detection of high frequency signals, the current methods are: secondary sampling, frequency shift by varying scale and modem [24, 25], and so on. The main idea is transform the high frequency into the low frequency through the scale change to meet the low frequency of the small parameter conditions, so that it is able to generate stochastic resonance effect. Finally, the frequency of the output signal recover its actual measurement scale, which is the frequency of the signal to be measured. These methods have some inevitably problem of the efficiency and practicality.

(i) In the measurement of the actual engineering, such as mechanical failure diagnosis, most of the signal to be measured is the high-frequency signal, and the noise is often colored noise, rather than idealized Gaussian white noise.

(ii) In the field of classical stochastic resonance, most theoretical studies only discuss the linear response of single frequency weak signal, and it can be observed clearly that the output signal of stochastic resonance system has some distortion. Compared to the original 


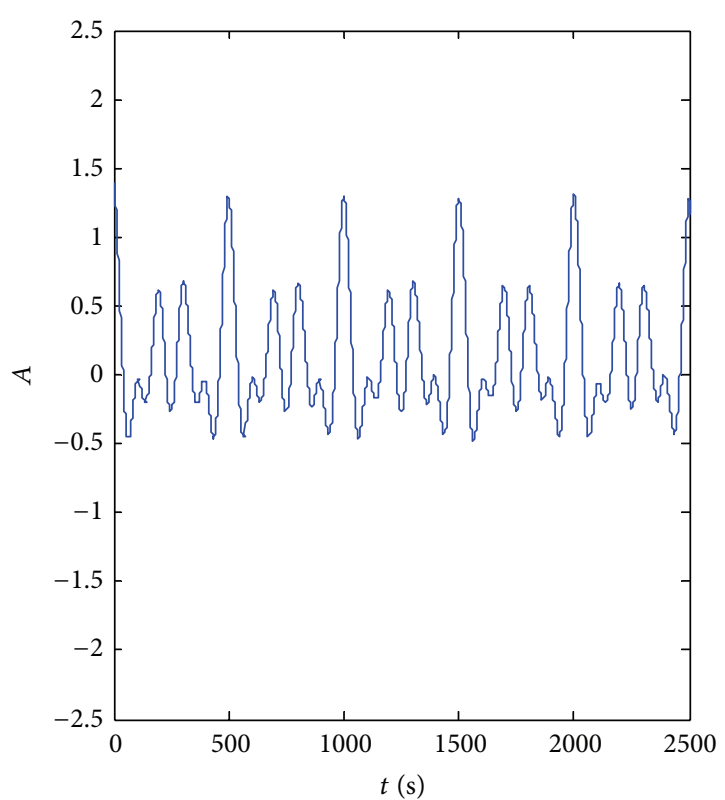

(a)

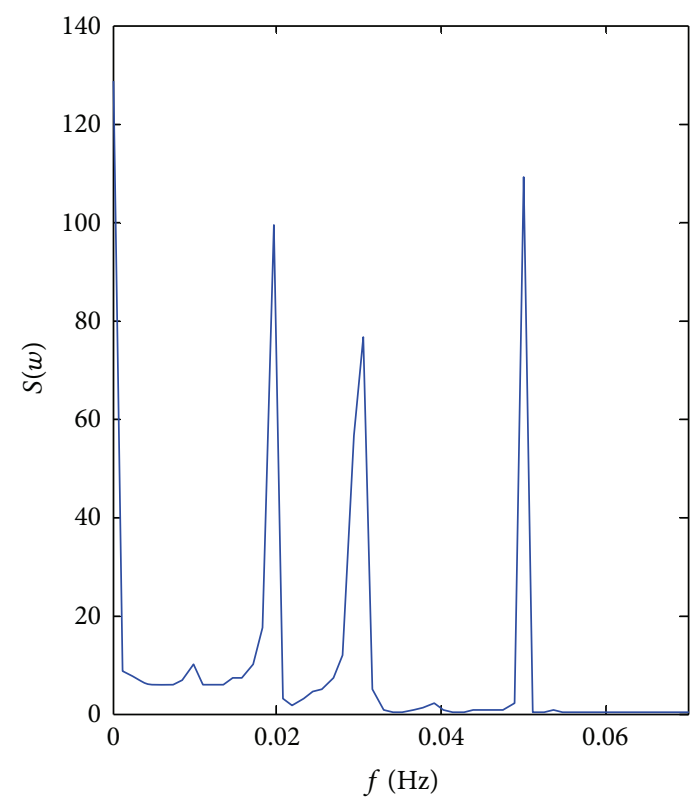

(b)

Figure 7: (a) The time-domain diagram of stochastic resonance output signal after correlation processing. (b) The spectrum diagram of stochastic resonance output signal after correlation processing.

sinusoidal signal, the output signal is more similar to a rectangular wave. Depending on the nature of the rectangular wave, the Fourier expansion is

$$
x(t)=\frac{4 A}{\pi}\left(\sin \omega_{0} t+\frac{1}{3} \sin 3 \omega_{0} t+\frac{1}{5} \sin 5 \omega_{0} t+\cdots\right) .
$$

Except for the fact that the $\omega_{0}$ has peak, its odd multiples of frequency $3 \omega_{0}, 5 \omega_{0} \ldots$ have peaks in the spectrum diagram of the system output signal. Taking into account the influence of noise, the signal to be measured with noise meet is Lorentz distribution through the stochastic resonance system, and the odd multiples of the output signal frequency are not obvious in the spectrum diagram. However, in the detection of actual signals, the measured signal may exist with multifrequencies, and satisfy the relationship of odd multiple, and it is difficult to determine the frequency which, corresponding to the peak, is the frequency of the output signal or some other weak signals by nonlinear response. Therefore, the method of low-frequency signal detection is not suitable for it and it needs to make some adjustments. A method is proposed for the above problems in this paper, which is approaching constantly the frequency of the signal to be measured by automatically adjusting the modulation signal frequency $f_{c}$ of the system externally added, and thereby detecting the frequency of the signal being measured. The main idea is as follows.

Let the input signal be measured as

$$
s(t)=\sum_{i=1}^{M} A_{i} \cos \left(2 \pi f_{i} t\right)+\Gamma(t),
$$

where $f_{i}(i=1,2, \ldots M)$ is the frequency of the signal to be measured. $\Gamma(t)$ is color noise distinguished from white Gaussian noise, and color noise is nonzero. Let its frequency mainly concentrate in some band of $0.2 \mathrm{~Hz}-0.5 \mathrm{~Hz}$ in this paper. Adding one cycle of the modulation signal to the system, the input signal to be measured is transformed into:

$$
\begin{aligned}
F(t) \cdot S(t)= & \frac{1}{2} \sum_{i=0}^{M} A_{i} \cos \left[2 \pi\left(f_{i}-f_{c}\right)\right] \\
& +\frac{1}{2} \sum_{i=1}^{M} A_{i} \cos \left[2 \pi\left(f_{i}-f_{c}\right)\right] \\
& +\Gamma(t) \cdot \cos \left(2 \pi f_{c} t\right) .
\end{aligned}
$$

The signal is composed of two parts: the difference frequency $f_{i}-f_{c}$, and the added frequency $f_{i}+f_{c}$.

It constantly approachs the frequency of the signal being measured $f_{i}$ by adjusting the frequency $f_{c}$ from $f_{c}<f_{i}$ via $f_{c}=f_{i}$ to $f_{c}>f_{i}$, difference frequency $f_{i}-f_{c} \ll 1$ which meets the generated conditions of the stochastic resonance in a certain frequency band. The system will generate a random resonance effect at this time, which means that each $f_{c}$ will exists with a significantly nonzero spectral peak corresponding to the output signal spectrogram. Particularly, while $f_{c}=f_{i}$, the stochastic resonance disappears. The maximum spectral peak power is close to 0 , and its reciprocal is infinite, which seems like a sharp peak in the diagram. So that we can use this feature to exacte the frequency of the input signals to be measured $f_{i}$. This method avoids the problem of odd multiples mentioned above. The frequency of the color noise is often concentrated in some frequency band. So it is difficult to distinguish the color noise and the frequency of the signal to be measured from the frequency domain. It is no longer applicable to use SNR as the index. 


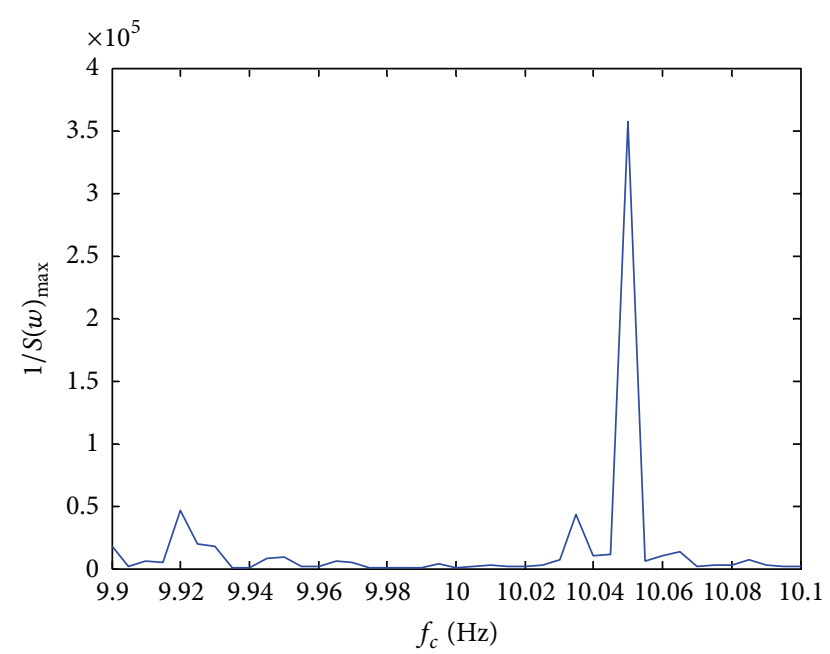

FIGURE 8: The change curve about the reciprocal of the stochastic resonance output signal spectrum peak with the adjustment of $f_{c}$ in the single high frequency.

This paper selects the reciprocal of the maximum power spectrum peak of the output signal the autocorrelation function as measurement index.

The steps of adaptive stochastic resonance in the highfrequency signal detection are as follows.

(a) Set the system parameters, select the appropriate value interval, and fix the step size $h=1 / f_{s}$. Increase the step size gradually to adjust $f_{c}$, approaching the frequency of the signal to be measured $f_{i}$.

(b) Make numerical simulation of each $f_{c}$ corresponding system by the fourth-order Runge-Kutta algorithm, and get the system output signal corresponding to each parameter points. Plott the curve of the maximum power spectral peak in the output signal with the modulating signal frequency $f_{c}$ changed.

(c) Sharp peaks will appear in the curve which is drawn above, and each frequency corresponding to the peak is the frequency of the signal to be measured $f_{i}$.

The flow chart is shown in Figure 10.

4.1. Simulation of the Single High-Frequency Signal Detector. Let the system parameters $a=1.4, b=1$, the signal to be measured is $s(t)=A \cos \left(2 \pi f_{0} t\right)$, while $A=2$, $f_{0}=10.05 \mathrm{~Hz}$, the color noise is generated by the MATLAB script. The sampling frequency is $f_{s}=5000$. The adjustment interval of the modulation frequency $f_{c}$ is [9.9 10.1]. Adjust the frequency $f_{c}$ to approach the frequency of the signal being measured $f_{i}$. As shown in Figure 8, it occurred a sharp peak while $f_{c}=10.05 \mathrm{~Hz}$, which means that the frequency of the signal being measured is $10.05 \mathrm{~Hz}$. The numerical simulation results comes together with the theoretical analysis, so this method is effective and feasible.

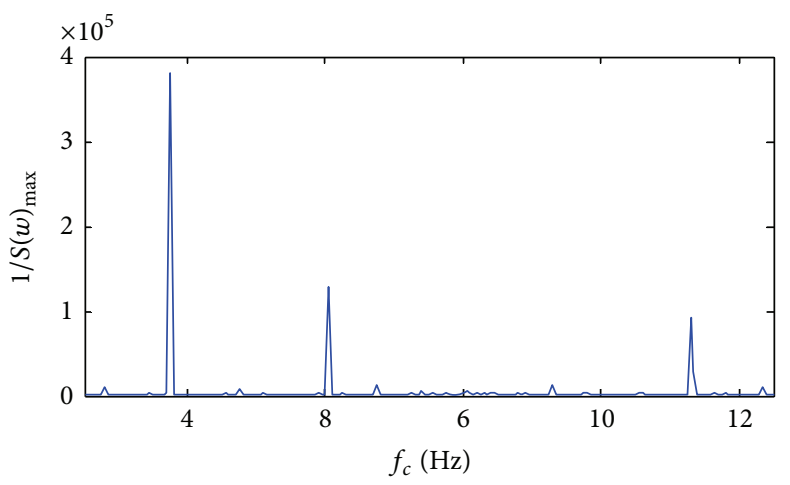

FIGURE 9: The change curve about the reciprocal of the stochastic resonance output signal spectrum peak with the adjustment of $f_{c}$ in the multiple high frequency.

4.2. Simulation of the Multiple High-Frequency Signal Detector. Let the input signal be detected with multiple high frequency as follows:

$$
s(t)=\sum_{i=1}^{3} A_{i} \cos \left(2 \pi f_{i} t\right)
$$

where the amplitude $A_{1}=2, A_{2}=1.5, A_{3}=2.1$, the frequency $f_{1}=3.75 \mathrm{~Hz}, f_{2}=6.05 \mathrm{~Hz}, f_{3}=11.30 \mathrm{~Hz}$, the bistable system parameters $a=1.3, b=1$, and the noise intensity $D=10$. Sampling frequency $f_{s}=5000$. The modulation signal frequency range is $[2.5,12.5]$. As shown in Figure 9 , the frequencies $f_{1}, f_{2}$, and $f_{3}$ all appear obvious as sharp peaks, it detected the frequency of the multiple signals submerged by strong noise efficiently. The odd multiples of the frequency $3 f_{1}$ are close to the frequency $f_{3}$. The simulation results show that the detected signal frequency is $f_{3}$ which is the frequency of the input signal to be measured rather than the odd multiples. It proves that the method is feasible, effective, and suitable for the actual engineering measurement.

\section{Conclusions}

In order to meet the needs of practical engineering, this paper combined the adaptive algorithm with stochastic resonance theory. According to the frequency characteristics of the input signal to be tested, it proposed a feasible and effective adaptive stochastic resonance signal detection. Considering the actual situation, it improves work efficiency to a certain extent and has great value and development prospects in the measurement of the actual engineering. This paper chooses the SNR and the power spectrum of the autocorrelation function estimates as the index. The characteristics of the signal to be measured contain a lot of complexity in practical applications. In the actual engineering, we can choose a more precise measurement of indicators to measure the generation of stochastic resonance effect. Among the system parameters, noise intensity and the frequency of the signal being measured, which have a close relationship. We can analyze the degree of association by genetic algorithm to 


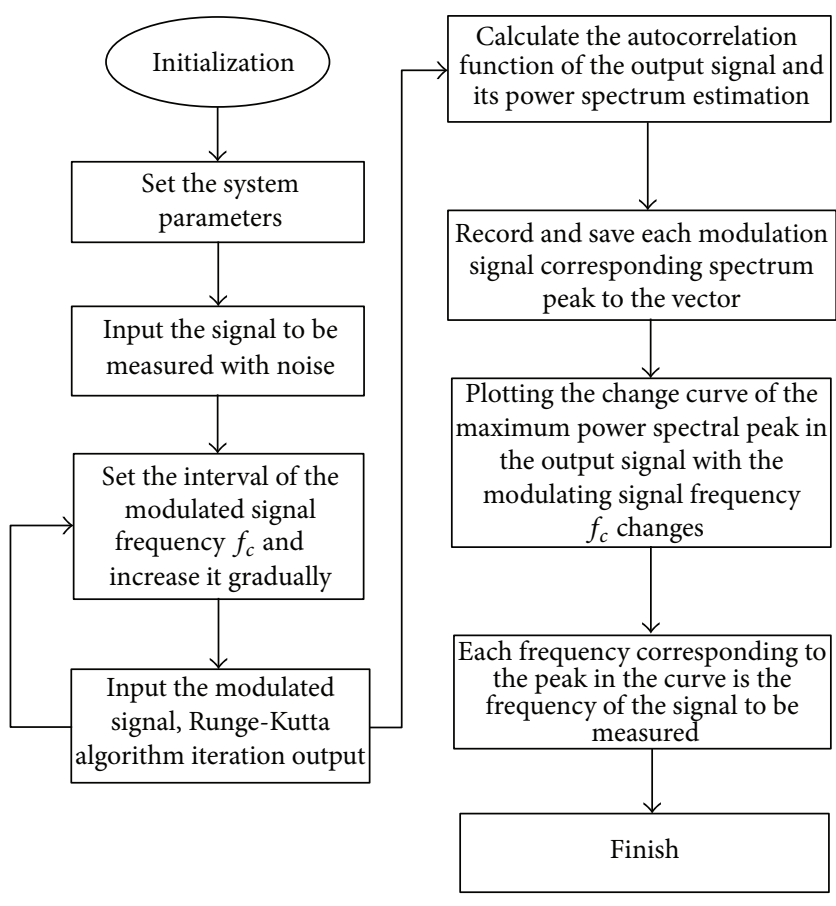

FIgure 10: The flow chart.

further expand the system of stochastic resonance signal detection.

\section{Acknowledgments}

This work was supported by National Natural Science Foundation of China (nos. 61104062 and 61174077), Jiangsu Qing Lan Project, and PAPD.

\section{References}

[1] M. Witzke, "Linear and widely linear filtering applied to iterative detection of generalized MIMO signals," Annales des Telecommunications, vol. 60, no. 2, pp. 113-117, 2005.

[2] R. Benzi, A. Sutera, and A. Vulpiani, "The mechanism of stochastic resonance," Journal of Physics A, vol. 14, no. 11, pp. L453L457, 1981.

[3] F. Duan and D. Abbott, "Binary modulated signal detection in a bistable receiver with stochastic resonance," Physica A, vol. 376, no. 1-2, pp. 173-190, 2007.

[4] C. Y. Chen, M. H. Ma, B. Zhao, S. F. Xie, and B. R. Xiang, "Stochastic resonance algorithm applied to quantitative analysis for weak liquid chromatographic signal of pyrene in water samples," International Journal of Environmental Analytical Chemistry, vol. 91, no. 1, pp. 112-119, 2011.

[5] W. Wei, X. Suyun, and X. Shaofei, "An adaptive single-well stochastic resonance algorithm applied to trace analysis of clenbuterol in human urine," Molecules, vol. 17, no. 2, pp. 19291938, 2012.

[6] L.-F. Li and J.-Y. Zhu, "Gravitational wave detection: stochastic resonance method with matched filtering," General Relativity and Gravitation, vol. 43, no. 11, pp. 2991-3000, 2011.
[7] F. Guo and Y. R. Zhou, "Stochastic resonance in a stochastic bistable system subject to additive white noise and dichotomous noise," Physica A, vol. 388, no. 17, pp. 3371-3376, 2009.

[8] H. Yan, W. Tai-yong, W. Jian, and Z. Pan, "Mechanical fault diagnosis based on the cascaded bistable stochastic resonance and multi-fractal," Journal of Vibration Shock, vol. 31, no. 8, pp. 181-185, 2012.

[9] P. Ping, Y. Ping, and H. Zhaoxia, "Speaker recognition method based on stochastic resonance," Telecommunications Science, vol. 26, no. S2, pp. 74-78, 2010.

[10] J. W. Mo, S. Ouyang, H. L. Xiao, and X. Y. Sun, "High sensitive GPS signal acquisition algorithm based on stochastic resonance," Systems Engineering and Electronics, vol. 33, no. 4, pp. 838-841, 2011.

[11] Z. S. Chen and Y. M. Yang, "Stochastic resonance mechanism for wideband and low frequency vibration energy harvesting based on piezoelectric cantilever beams," Acta Physica Sinica, vol. 60, no. 7, Article ID 074301, pp. 1-7, 2011.

[12] Y. G. Leng, "Mechanism of parameter-adjusted stochastic resonance based on Kramers rate," Acta Physica Sinica, vol. 58, no. 8, pp. 5196-5200, 2009.

[13] G. Q. Zhu, K. Ding, Y. Zhang, and Y. Zhao, "Experimental research of weak signal detection based on the stochastic resonance of nonlinear system," Acta Physica Sinica, vol. 59, no. 5, pp. 3001-3006, 2010.

[14] Y. Hasegawa and M. Arita, "Escape process and stochastic resonance under noise intensity fluctuation," Physics Letters A, vol. 375, no. 39, pp. 336-372, 2011.

[15] H. Gang, G. Nicolis, and C. Nicolis, "Periodically forced FokkerPlanck equation and stochastic resonance," Physical Review A, vol. 42, no. 4, pp. 2030-2041, 1990.

[16] L. Yonggang and W. Tai-yong, "Numerical research of twice sampling stochastic resonance for the detection of a weak signal submerged in a heavy Noise," Acta Physica Sinica, vol. 52, no. 10, pp. 2432-2437, 2003.

[17] F. Chunfeng, G. Ke, C. Xin-wu, and G. Jiantao, "Application of frequency-shifted and re-scaling adaptive stochastic resonance in signal detection," Journal of Xinyang Normal University, vol. 23, no. 3, pp. 415-419, 2010.

[18] Q. Mao, M. Lin, and Y. Zheng, "Study of weak multi-frequencies signal detection based on stochastic resonance," Journal of Basic Science and Engineering, vol. 16, no. 1, pp. 86-91, 2008.

[19] Y. G. Leng, T. Y. Wang, Y. Guo, and Z. Y. Wu, "Study of the property of the parameters of bistable stochastic resonance," Acta Physica Sinica, vol. 56, no. 1, pp. 30-35, 2007.

[20] J. F. Wang, F. Liu, J. Y. Wang, G. Chen, and W. Wang, "Frequency characteristics of the input thresholds of stochastic resonant systems," Acta Physica Sinica, vol. 46, no. 12, pp. 2311-2312, 1997.

[21] X. Jingsong, Weak Signal Deteetion Based on the theory of Stoehastic Resonance, Lanzhou University Of Information and Communication Engineering, Gansu, China, 2008.

[22] G. L. Zhang and F. Z. Wang, "Research of multiple signals in stochastic resonance system," Journal of System Simulation, vol. 21, no. 13, pp. 4190-4193, 2009.

[23] Y. G. Leng, T. Y. Wang, X. D. Qin, R. X. Li, and Y. Guo, "Power spectrum research of twice sampling stochastic resonance response in a bistable system," Acta Physica Sinica, vol. 53, no. 3, pp. 717-723, 2004.

[24] Y. G. Leng, Y. S. Leng, T. Y. Wang, and Y. Guo, "Numerical analysis and engineering application of large parameter stochastic 
resonance," Journal of Sound and Vibration, vol. 292, no. 3-5, pp. 788-801, 2006.

[25] Y. Dingxin, H. Zheng, and Y. Yongmin, "The analysis of stochastic resonance of periodic signal with large parameters," Acta Physica Sinica, vol. 61, no. 8, Article ID 080501, 2012. 


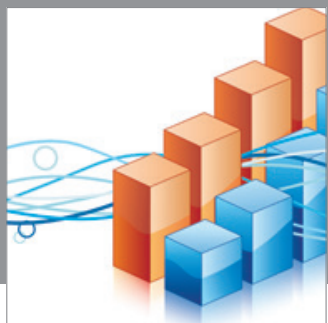

Advances in

Operations Research

mansans

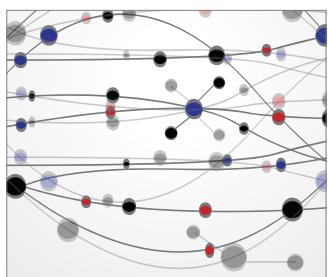

The Scientific World Journal
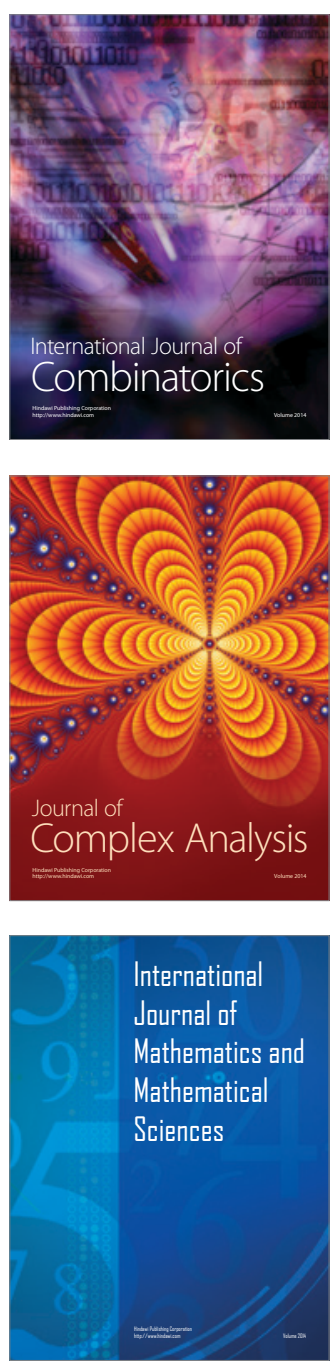
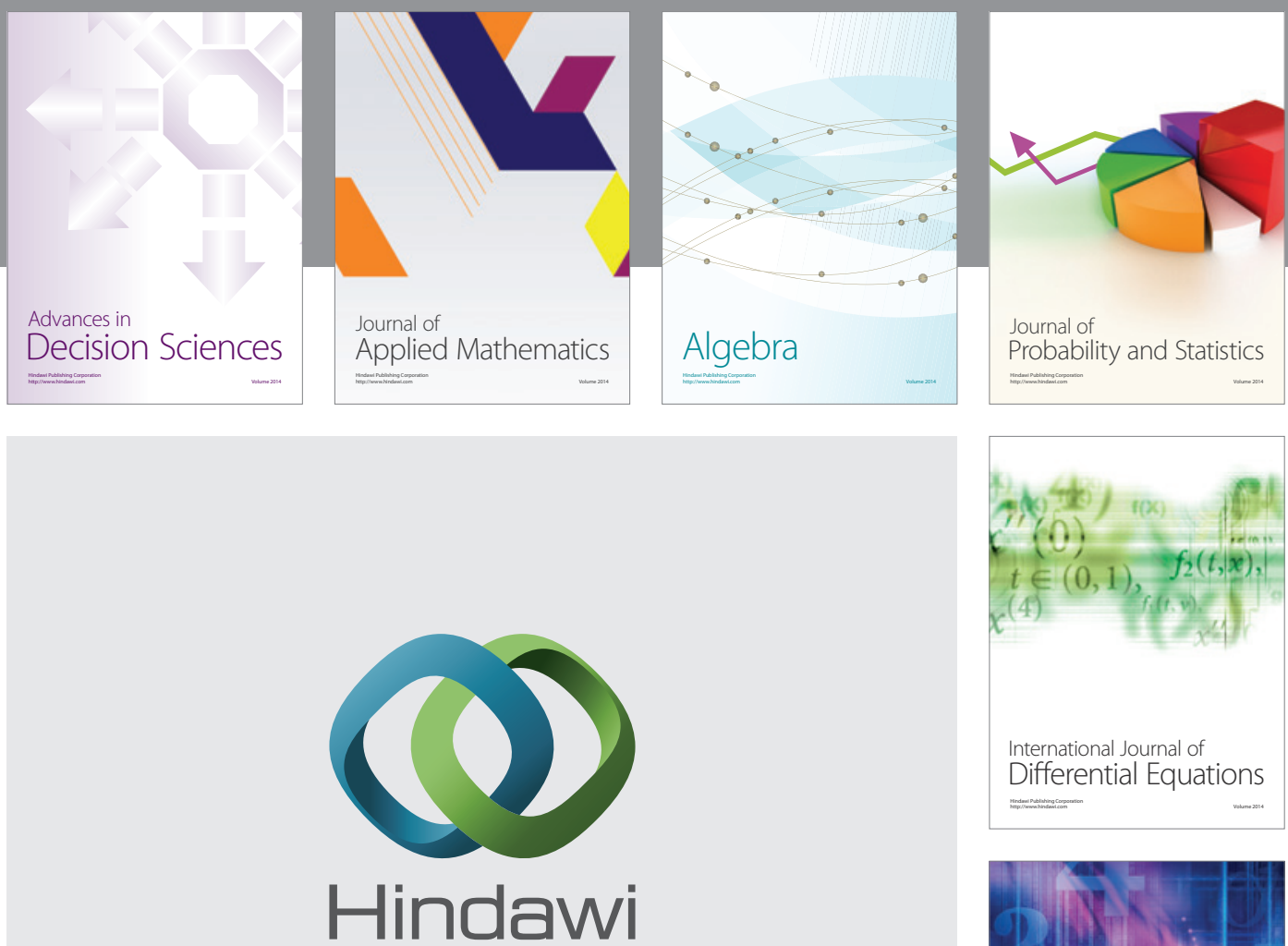

Submit your manuscripts at http://www.hindawi.com
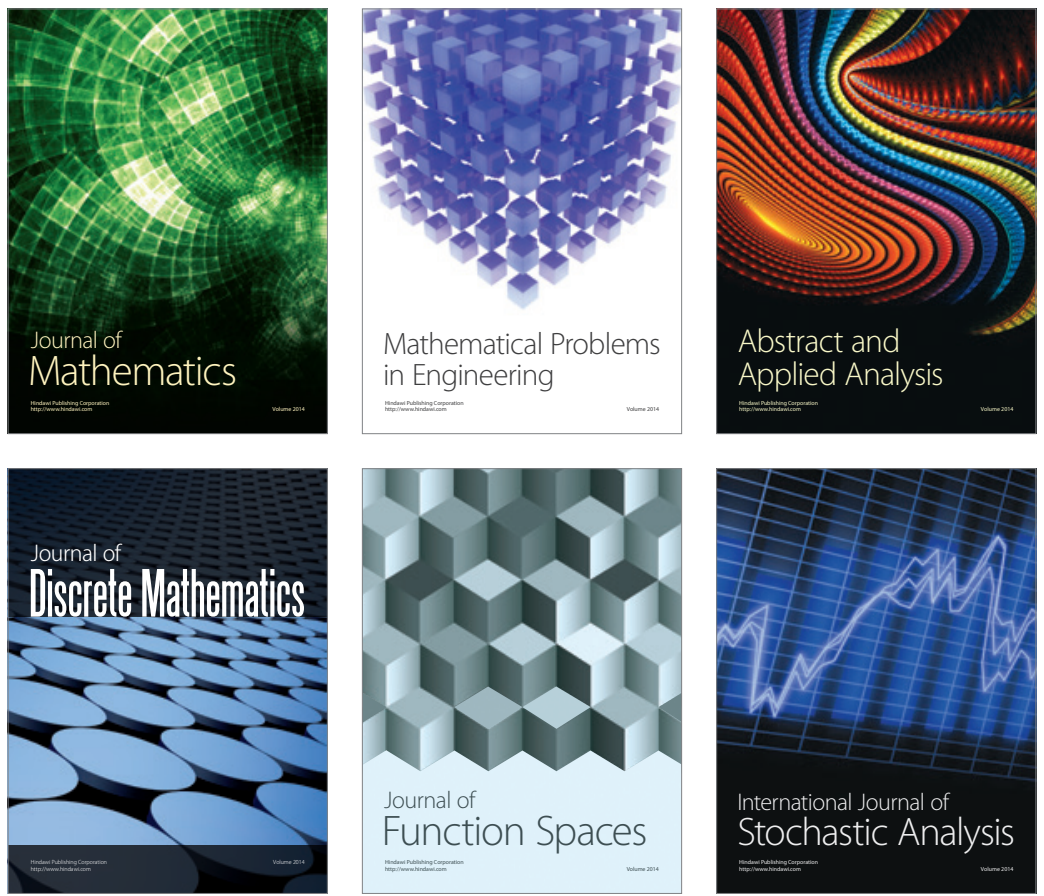

Journal of

Function Spaces

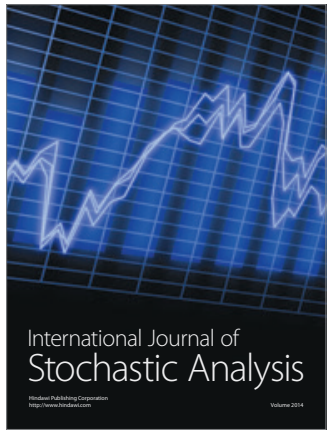

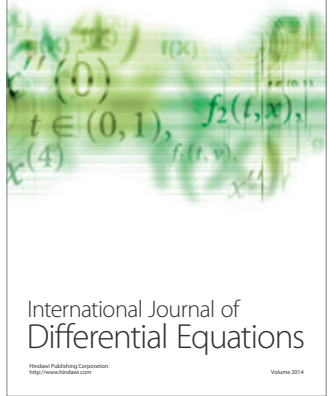
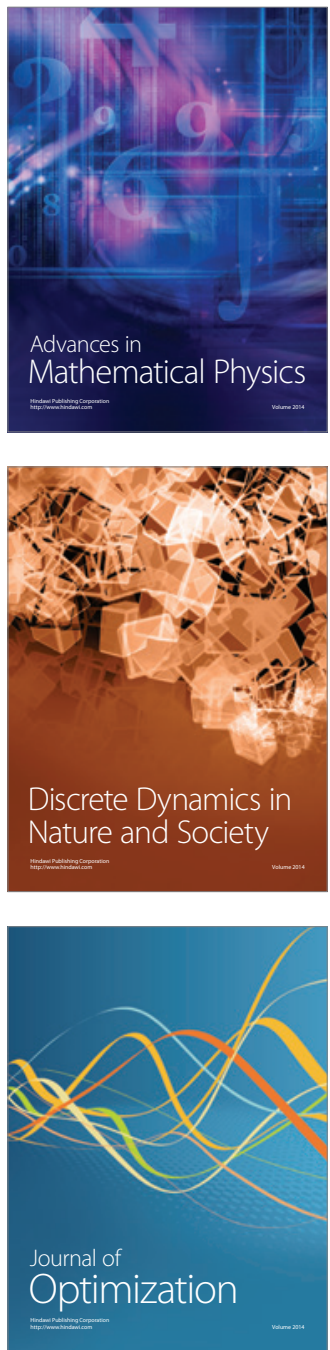